\title{
Implication of atypical supraclavicular F18-fluorodeoxyglucose uptake in patients with breast cancer: Association between brown adipose tissue and breast cancer
}

\author{
TAKAAKI FUJII, REINA YAJIMA, HIRONORI TATSUKI, KATSUYA OOSONE and HIROYUKI KUWANO \\ Department of General Surgical Science, Graduate School of Medicine, \\ Gunma University, Maebashi, Gunma 371-8511, Japan
}

Received October 9, 2015; Accepted February 23, 2017

DOI: $10.3892 / \mathrm{ol} .2017 .6768$

\begin{abstract}
It has been reported that F18-fluorodeoxyglucose (FDG) uptake in the neck and supraclavicular lesions represents activated brown adipose tissue (BAT). In the present study, the association between BAT activity, detected by FDG-positron emission tomography (PET), and the clinicopathological features of patients with breast cancer was investigated. The cases of 156 consecutive patients with breast cancer who underwent FDG-PET preoperatively were analyzed. The distribution and intensity of atypical FDG uptake in the neck and/or supraclavicular region was reviewed. The intensity was graded as follows: 1, weak; 2, moderate; and 3, intense. Among the 156 patients, 70 (44.9\%) exhibited grade 1 intensity, $65(41.7 \%)$ exhibited grade 2 intensity and $21(13.5 \%)$ exhibited grade 3 intensity. The intensity of FDG was significantly associated with human epidermal growth factor receptor 2 (HER2) expression and progesterone expression. Among the 156 patients, 6 (3.8\%) had recurrent disease. Multivariate analysis revealed that showing a low grade of atypical FDG uptake was the only independent risk factor of short-term recurrence, and none of the patients with recurrent disease had atypical FDG uptake that may reflect the activation of BAT. These results indicated that the presence of BAT is associated with HER2 expression and the absence of BAT may be a prognostic factor for breast cancer.
\end{abstract}

\section{Introduction}

In recent years, the clinical applications of positron emission tomography (PET) have undergone explosive growth (1-6). PET using F18-fluorodeoxyglucose (FDG) is a noninvasive

Correspondence to: Dr Takaaki Fujii, Department of General Surgical Science, Graduate School of Medicine, Gunma University, 3-39-22 Showa-machi, Maebashi, Gunma 371-8511, Japan

E-mail: ftakaaki@gunma-u.ac.jp

Key words: brown adipose tissue, F18-fluorodeoxyglucose-positron emission tomography, breast cancer whole-body imaging technique used to evaluate various types of malignancies, including breast cancer, and for tumor staging, tumor restaging, the detection of recurrence and monitoring treatment responses (1-6). However, FDG uptake is not tumor-specific, and PET imaging also provides quantitative information about numerous diseases, including inflammation and infection (7). Several studies have reported cases of neck and supraclavicular FDG uptake that were not associated with any radiologically or clinically detectable pathology (8-10). Atypical neck and supraclavicular FDG uptake is not an uncommon finding and may be demonstrated to partly represent brown adipose tissue (BAT) (10-12). BAT activation helps maintain normal body temperatures in newborns (13-15). Although the amount of BAT declines with age, islets of brown adipocytes remain in the white adipose tissue of adult humans (13-15). BAT has recently attracted attention, since it consumes stored energy and may thereby be involved in obesity and age-associated metabolic disease (15-17). Due to the similarity of its biological property of hypermetabolism to cancer cells, BAT may also show intense FDG uptake on FDG-PET imaging (15-17). It has been reported that a high level of BAT in adult humans is associated with cancer-induced cachexia and may reflect an abnormality in a mechanism responsible for substantial energy expenditure $(15,18)$. BAT has recently emerged as a topic of focus in the context of cancer and tumor development (19-21). However, data on the association of BAT and cancer are limited. In the present study, the association between BAT activity detected by FDG-PET and clinicopathological features in cases with primary breast cancer was examined.

\section{Patients and methods}

Patient information and data analysis. The present study retrospectively investigated 156 female patients with primary breast cancer who underwent FDG-PET preoperatively at the Department of General Surgical Science, Gunma University (Gunma, Japan) between January 2010 and September 2014. All the patients had undergone radical breast surgery. Patients with previously diagnosed breast cancer or incomplete clinical information were excluded. Additionally, male patients were 
A
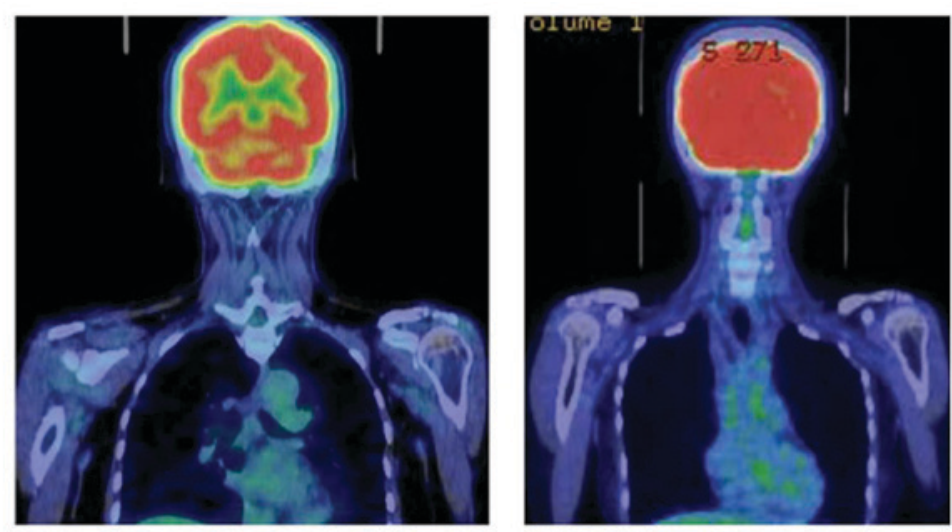

B
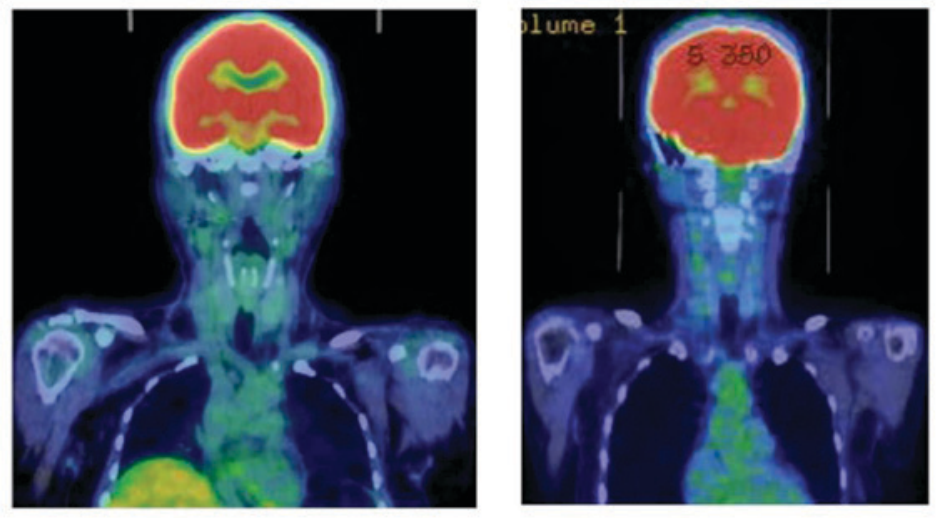

C
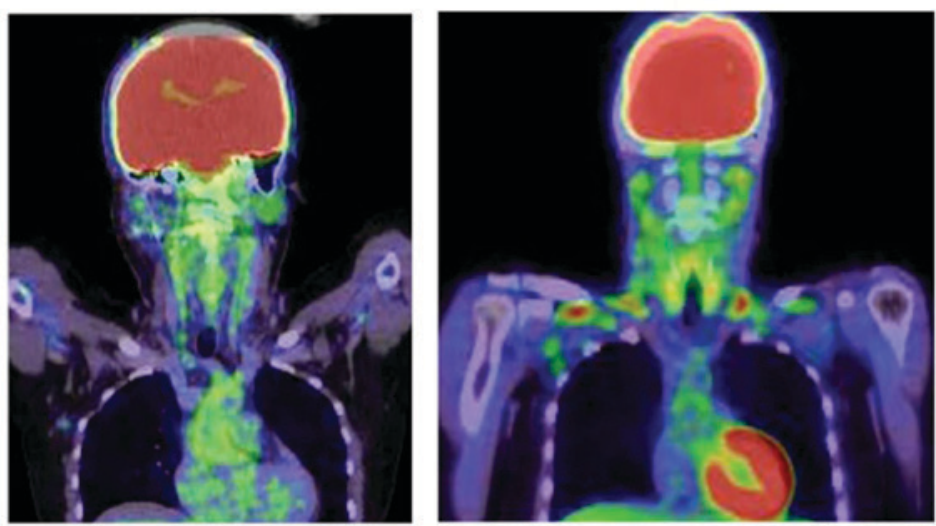

Figure 1. In patients with atypical F18-fluorodeoxyglucose uptake in the neck and/or supraclavicular region, the intensity was graded as follows: (A) 1, weak; (B) 2 , moderate; and (C) 3, intense.

excluded. The mean age of the patients was $58.6 \pm 12.3$ years, with an age distribution between 32 and 86 years. Patients underwent their FDG-PET/CT as part of the routine standard of care $(5,6)$, and no changes to the standard of care were made. The maximum standardized uptake value $\left(\mathrm{SUV}_{\max }\right)$ of primary tumors was calculated in a routine clinical fashion. Written informed consent was obtained from all patients for the use of their records and imaging in future studies, and this was approved by the Clinical Ethics Committee of Gumma University (Gumma, Japan).

The distribution and intensity of BAT was reviewed in all patients in the present study. Images were first assessed visually for any atypical, non-pathological increased uptake in the neck and/or supraclavicular region. Atypical uptake was defined as lesions that exhibited an increased focal or linear FDG uptake bilaterally in the neck and/or supraclavicular region. In patients with atypical FDG uptake in the neck and/or supraclavicular region, the intensity scores were graded as follows: 1, weak; 2, moderate; and 3, intense (Fig. 1).

The details extracted from the database were age, histological type, primary tumor size, nuclear grade, lymph node metastasis, lymphatic or vascular invasion, estrogen (ER) or progesterone $(\mathrm{PgR})$ status, human epidermal growth factor receptor 2 (HER2) expression and $\mathrm{SUV}_{\max }$ of the primary tumor, serum tumor markers [carcinoembryonic antigen, (CEA)] and body mass index (BMI). The ER and PgR statuses were assessed by ALLRED scores $(22,23)$. ALLRED scores are calculated by adding two numbers, one reflecting the proportion of positive tumor cells $(0$, none; $1,<1 \% ; 2,1-10 \%$; $3,11-33 \%$; 4, 34-66\%; and 5, 67-100\%) and the other reflecting the intensity of immunoreactivity (1, weak; 2 , moderate; 3 , strong). The maximum score is 8 and an ALLRED score of 
Table I. Patient characteristics and clinicopathological features associated with atypical supraclavicular FDG-uptake.

FDG-uptake in neck/supraclavicular region, $\mathrm{n}$

\begin{tabular}{|c|c|c|c|c|}
\hline \multirow{2}{*}{ Characteristic } & & \multirow[b]{2}{*}{ P-value } \\
\hline & Grade $1(n=70)$ & Grade $2(n=65)$ & Grade $3(n=21)$ & \\
\hline Age, years ${ }^{\mathrm{a}}$ & $58.2 \pm 13.0$ & $58.8 \pm 11.4$ & $59.1 \pm 11.0$ & 0.940 \\
\hline Postmenopausal & 49 & 19 & 15 & 0.519 \\
\hline Tumor size, $\mathrm{mm}^{\mathrm{a}}$ & $22.5 \pm 18.7$ & $20.3 \pm 15.8$ & $18.0 \pm 12.3$ & 0.572 \\
\hline Histological type & & & & 0.428 \\
\hline Invasive ductal carcinoma & 55 & 54 & 19 & \\
\hline Ductal carcinoma in situ & 3 & 5 & 1 & \\
\hline Others & 12 & 6 & 1 & \\
\hline SUV $_{\max }$ of primary tumor ${ }^{\mathrm{a}}$ & $4.0 \pm 5.4$ & $3.5 \pm 3.4$ & $2.3 \pm 1.7$ & 0.219 \\
\hline Lymph node metastasis & 18 & 16 & 4 & 0.821 \\
\hline ER-positive & 62 & 54 & 14 & 0.061 \\
\hline PgR-positive & 60 & 50 & 11 & 0.006 \\
\hline HER2-positive & 1 & 12 & 12 & $<0.001$ \\
\hline Nuclear grade 3 & 22 & 23 & 7 & 0.888 \\
\hline Lymphatic invasion & 26 & 22 & 9 & 0.750 \\
\hline Vascular invasion & 11 & 12 & 1 & 0.314 \\
\hline CEA, $<3.0$ & 15 & 11 & 5 & 0.717 \\
\hline $\mathrm{BMI}, \mathrm{kg} / \mathrm{m}^{2}$ a & $22.5 \pm 4.6$ & $23.1 \pm 3.0$ & $24.4 \pm 3.6$ & 0.146 \\
\hline
\end{tabular}

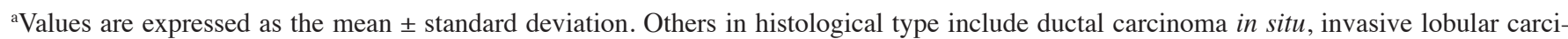
noma, mucinous carcinoma and medullary carcinoma. $\mathrm{SUV}_{\max }$, maximum standardized uptake value; ER, estrogen receptor; PgR, progesterone receptor; CEA, carcinoembryonic antigen; BMI, body mass index; HER2, human epidermal growth factor receptor 2.

$\geq 3$ was defined as ER- and PgR-positive $(22,23)$. The overall median follow-up period was 21.8 months, and none of the patients died of surgical complications.

Statistical analysis. The breast cancer cases were divided into 3 groups on the basis of the intensity grade of the atypical FDG uptake in the neck and/or supraclavicular region. All data were analyzed by one-way analysis of variance with Fisher's adjustment. The breast cancer cases were then divided into two groups on the basis of the presence or absence of recurrence. A univariate statistical analysis was conducted using Fisher's exact test or the $\chi^{2}$ test with or without Yates' correction. To compare the two groups, Student's t-test was performed. To test the independence of the risk factors, the variables were entered into a multivariate logistic regression model with a likelihood of $\mathrm{P}<0.05$. The relapse-free survival (RFS) was calculated using the Kaplan-Meier method. The log-rank test was used to evaluate the differences between the recurrence-free intervals. $\mathrm{P}<0.05$ was considered to indicate a statistically significant difference.

\section{Results}

Atypical neck and supraclavicular FDG uptake is associated with HER2 and PgR expression in patients with breast cancer. The 156 consecutive patients with breast cancer who underwent FDG-PET preoperatively were analyzed. The patients with breast cancer were divided into 3 groups based on the intensity of atypical neck and/or supraclavicular FDG uptake. Among the 156 patients, $70(44.9 \%)$ patients were grade 1, $65(41.7 \%)$ were grade 2 and 21 (13.5\%) were grade 3. Table I summarizes not only the patient characteristics but also the results of the analysis conducted to determine the association between the atypical FDG uptake and clinicopathological variables. The analysis revealed that HER2 expression $(\mathrm{P}<0.001)$ and $\mathrm{PgR}$ expression $(\mathrm{P}=0.006)$ were statistically significant factors. The tumor size and $\mathrm{SUV}_{\max }$ of the primary tumor were relatively increased in patients with high intense atypical FDG uptake, compared with that in patients with weak or moderate atypical FDG uptake; although, no statistically significant difference was identified. Average age and BMI were not statistically significant factors in the current study.

Atypical FDG uptake is associated with short-term disease recurrence in breast cancer. The patients with breast cancer were divided into 2 groups based on the presence of recurrence. Among the 156 patients, 6 (3.8\%) exhibited recurrent disease. Table II summarizes not only the patient characteristics but also the results of the univariate analysis conducted to determine the association between the clinicopathological variables and recurrent disease. The univariate analysis revealed that vascular invasion $(\mathrm{P}=0.047)$ and a low grade of atypical FDG uptake $(\mathrm{P}=0.022)$ were statistically significant factors. The multivariate analysis revealed that only a low grade of atypical FDG uptake $(\mathrm{P}=0.038)$ was an independent risk factor of short-term recurrence. Vascular invasion $(\mathrm{P}=0.079)$ lost its significance in the multivariate analysis. 
Table II. Patient characteristics and clinicopathological features associated with recurrent disease.

\begin{tabular}{|c|c|c|c|}
\hline \multirow[b]{2}{*}{ Characteristic } & \multicolumn{2}{|c|}{ Recurrence, $\mathrm{n}$} & \multirow[b]{2}{*}{ P-value } \\
\hline & Negative $(n=150)$ & Positive $(\mathrm{n}=6)$ & \\
\hline \multicolumn{4}{|l|}{ Atypical FDG-uptake } \\
\hline Grade 1 & 64 & 6 & 0.022 \\
\hline Grade 2 & 65 & 0 & \\
\hline Grade 3 & 21 & 0 & \\
\hline Age, years & $58.6 \pm 12.5$ & $59.3 \pm 7.5$ & 0.902 \\
\hline Postmenopausal & 110 & 5 & 0.501 \\
\hline Tumor size, $\mathrm{mm}^{\mathrm{a}}$ & $20.7 \pm 16.9$ & $27.4 \pm 12.8$ & 0.164 \\
\hline \multicolumn{4}{|l|}{ Histological type } \\
\hline Invasive ductal carcinoma & 124 & 4 & 0.428 \\
\hline Ductal carcinoma in situ & 9 & 0 & \\
\hline Others & 17 & 2 & \\
\hline $\mathrm{SUV}_{\max }$ of primary tumor ${ }^{\mathrm{a}}$ & $3.5 \pm 4.4$ & $4.3 \pm 2.3$ & 0.331 \\
\hline Lymph node metastasis & 36 & 2 & 0.845 \\
\hline ER-positive & 125 & 5 & 0.738 \\
\hline PgR-positive & 116 & 5 & 0.505 \\
\hline HER2-positive & 25 & 0 & 0.344 \\
\hline Nuclear grade 3 & 49 & 3 & 0.371 \\
\hline lymphatic invasion & 53 & 4 & 0.130 \\
\hline vascular invasion & 21 & 3 & 0.047 \\
\hline CEA $(3.0<)$ & 29 & 2 & 0.907 \\
\hline Adjuvant hormone therapy & 116 & 5 & 0.595 \\
\hline Adjuvant chemotherapy & 46 & 3 & 0.321 \\
\hline Adjuvant HER2-targeted therapy & 21 & 0 & 0.414 \\
\hline BMI $\left(\mathrm{kg} / \mathrm{m}^{2}\right)$ & $24.4 \pm 3.6$ & $25.1 \pm 5.2$ & 0.188 \\
\hline
\end{tabular}

${ }^{a}$ Values are expressed as the mean \pm standard deviation. Others in histological type include ductal carcinoma in situ, invasive lobular carcinoma, mucinous carcinoma and medullary carcinoma. $\mathrm{SUV}_{\max }$, maximum standardized uptake value; ER, estrogen receptor; PgR, progesterone receptor; CEA, carcinoembryonic antigen; BMI, body mass index; HER2, human epidermal growth factor receptor 2.

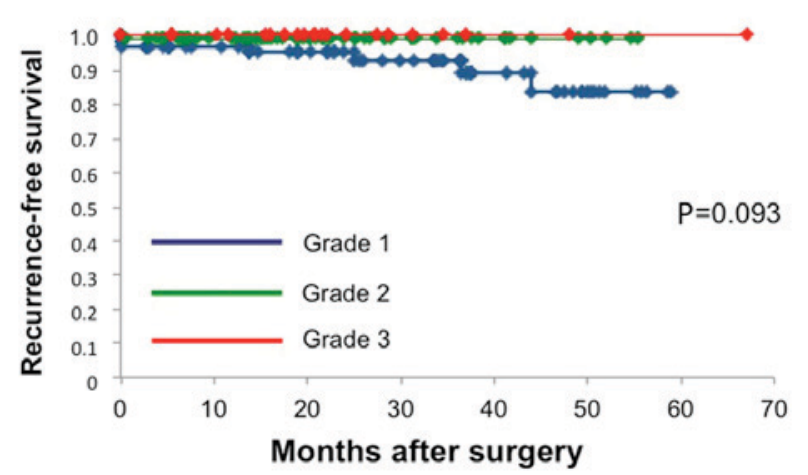

Figure 2. The recurrence-free survival shown by Kaplan-Meier curves was relatively shorter for the patients with grade 1 atypical FDG uptake, compared with those with grade 2 and 3 , but no statistically significant differences were observed.

The RFS shown by the Kaplan-Meier curves was relatively shorter for the patients with grade 1 atypical FDG uptake, compared with those with grade 2 and 3, but no statistically significant differences were observed (Fig. 2).

\section{Discussion}

FDG-PET has been widely used for staging and identifying recurrence in various types of cancer (1-6). Neck and supraclavicular FDG uptake is not a frequent phenomenon in the general PET population (8-12). Previous studies have reported that FDG uptake in neck and supraclavicular lesions represents activated BAT (10-12). Other studies reported such increased FDG uptake in BAT at a frequency of $2.3-4 \%$ of patients $(8-10,24,25)$. However, a high prevalence of activated BAT has been reported in patients with lymphoma (17\%) or breast cancer (16.7-80\%) among limited numbers of patients $(9,15,21,26)$. In the present study, atypical FDG uptake, which may represent active BAT, was detected in 86 of 156 patients (55.1\%), and intense FDG uptake was identified in 21 cases $(13.5 \%)$. In patients with cancer-induced cachexia, the prevalence of BAT was increased compared with in age-matched controls $(15,18)$. These results indicated an evident association between BAT and cancer status.

In the present study, the presence of atypical FDG uptake in neck and/or supraclavicular lesions was an independent risk factor for short-term disease recurrence, and all the patients 
with recurrent disease had atypical FDG uptake, which may reflect activated BAT. These results indicated that the presence of BAT may be considered an indicator of a lower level of biological aggressiveness and may be a prognostic factor in breast cancer. The mechanisms of the potential association between BAT and breast cancer have not been fully evaluated. There are two alternate hypotheses: BAT may be actively involved in the progression of breast cancer; and BAT may be passively involved in the progression of breast cancer, and the activation of BAT may be secondary to the breast cancer $(21,27,28)$. Notably, despite the presence of HER2 expression and a lower level of $\mathrm{PgR}$ expression, which are negative prognostic factors, the patient group in the present study with BAT included no patients with recurrent disease. It may appear that a paradoxical phenomenon was observed in the present study. However, breast cancer progression is a complex process, and it is regulated by multiple factors in addition to HER2 or PgR expression (29). These results indicated that disease recurrence may also occur via other mechanisms associated with BAT. Furthermore, HER2-positive breast cancer in the setting of HER2-targeted therapy is no longer associated with poor prognosis (30). In the current study, HER2-targeted therapy was performed in $82.2 \%$ patients with HER 2 positive breast cancer with grade 3 atypical FDG uptake. Additional study is necessary to substantiate the effect of BAT on breast cancer progression.

In the past several years, interest in subsets of obesity-associated tumors, including breast cancer, has increased in the field of cancer research (31-33). Increased fatty acid synthesis, which contributes to energy homeostasis and tumor growth, is a common feature of human tumors (32). The nuclear receptor peroxisome proliferation activated receptor $\gamma(\operatorname{PPAR} \gamma)$, a transcriptional master regulator of lipid metabolism, inhibits the growth of several common cancers (33). An acetylation-defective PPAR $\gamma$ mutant induces a brown phenotype in white adipocytes (33). The survival of breast cancer cells, particularly those with HER2 overexpression, is highly dependent on the lipid metabolism induced by PPAR $\gamma(34,35)$. Another study reported that cancer cell proliferation was inhibited by PPAR $\gamma$ mediated by lipid metabolism (31). Furthermore, in patients with cancer-induced cachexia, PPAR $\gamma$ serves important roles in the inflammation and inhibition of adipocyte differentiation (36). These findings, in combination with the present results, indicate the possibility that PPAR $\gamma$ may perform important roles in breast cancer progression and HER2 expression. Additional study is required to explore the mechanisms of breast cancer progression associated with PPAR $\gamma$ and BAT.

In the current study, PgR expression was associated with the presence of BAT. Previous studies have proposed that BAT metabolism is sex-dependent $(9,37,38)$. Testosterone was shown to inhibit the thermogenic response of BAT, and FDG uptake in BAT was revealed to be high in non-menopausal female patients $(9,37,38)$, which indicated that female sex hormones promote FDG uptake in BAT.

The present study had certain limitations, the primary one being that it was a retrospective analysis and the number of cases was relatively small. Furthermore, there are other known predictors or plausible factors associated with BAT activation $(18,19)$ that were not evaluated in the present study, including environmental temperature, psychological factors and catecholamine levels. FDG uptake in BAT has recently been reported to occur more commonly in weight-loss patients $(18,19)$. However, other studies also reported no significant difference between the BMI of patients with BAT $(18,19)$, which is consistent with our results. Additional investigation with a large number of patients is required to evaluate the clinical significance of BAT activation as detected by FDG-PET. However, the clinical implications of the data obtained in the present study are important, and to the best of our knowledge, this is the first study describing the clinical features and risk of recurrence of breast cancer associated with BAT.

In conclusion, the presence of atypical FDG uptake in neck and/or supraclavicular lesion, which may represent active BAT, was associated with HER2 and PgR expressions, and was an independent prognostic factor. These results may represent an evident association between BAT and cancer status. Additional study is required to explore the mechanisms of breast cancer progression, particularly the possibility of an association between PPAR $\gamma$ and BAT.

\section{Acknowledgements}

The authors would like to thank Y. Saitoh, T. Yano, Y. Matsui, A. Ishida and A. Ishiubo for their secretarial assistance. The present study was supported by Grants-in-Aid from the Japanese Ministry of Education, Culture, Sports, Science and Technology (grant no. 17K10534).

\section{References}

1. Kresnik E, Gallowitsch HJ, Mikosch P, Stettner H, Igerc I, Gomez I, Kumnig G and Lind P: Fluorine-18-fluorodeoxyglucose positron emission tomography in the preoperative assessment of thyroid nodules in an endemic goiter area. Surgery 133: 294-299, 2003.

2. Liu Y: Clinical significance of thyroid uptake on F18-fluorodeoxyglucose positron emission tomography. Ann Nucl Med 23: 17-23, 2009.

3. Fletcher JW, Djulbegovic B, Soares H, Siegel BA, Lowe VJ, Lyman GH, Coleman RE, Wahl R, Paschold JC, Avril N, et al: Recommendations on the use of F18-FDG PET in oncology. J Nucl Med 49: 480-508, 2008.

4. O'Connor OJ, McDermott S, Slattery J, Sahani D and Blake MA: The use of PET-CT in the assessment of patients with colorectal carcinoma. Int J Surg Oncol 2011: 846512, 2011.

5. Fujii T, Yajima R, Yamaguchi S, Tsutsumi S, Asao T and Kuwano H: Is it possible to predict malignancy in cases with focal thyroid incidentaloma identified by 18F-Fluorodeoxyglucose positron emission tomography? Am Surg 78: 141-143, 2012.

6. Fujii T, Suto T, Kigure W, Morita H, Katoh T, Yajima R, Tsutsumi S, Asao T and Kuwano H: Clinicopathological features of second primary colorectal cancer incidentally identified by 18F-FDG-PET. Hepatogastroenterology 62: 599-601, 2015.

7. Cook GJ, Fogelman I and Maisey MN: Normal physiological and benign pathological variants of 18-fluoro-2-deoxyglucose positron-emission tomography scanning: Potential for error in interpretation. Semin Nucl Med 26: 308-314, 1996.

8. Coohade C, Osman M, Pannu HK and Wahl RL: Uptake in supraclavicular area fat ('USA-fat'): Description on 18F-FDG PET/CT. J Nucl Med 44: 170-176, 2003.

9. Rousseau C, Bourbouloux E, Campion L, Fleury N, Bridji Bm Chatal JF, Resche I and Campone M: Brown fat in breast cancer patients: Analysis of serial (18)F-FDG PET/CT scans. Eur J Nucl Med Mol Imaging 33: 785-791, 2006.

10. Cohade C, Mourtzikos KA and Wahl RL: "USA-fat": Prevalence is related to ambient outdoor temperature-evaluation with 18F-FDG PET/CT. J Nucl Med 44: 1267-1270, 2003. 
11. Pace L, Nicolai E, D'Amico D, Ibello F, Della Morte AM, Salvatore B, Pizzuti LM, Salvatore M and Soricalli A: Determinants of physiologic 18F-FDG uptake in brown adipose tissue in sequential PET/CT examinations. Mol Imaging Biol 13: 1029-1035, 2011.

12. Cronin CG, Prakash P, Daniels GH, Boland GW, Kalra MK, Halpern EF, Palmer EL and Blake MA: Brown fat at PET/CT: Correlation with patient characteristics. Radiology 263: 836-842, 2012.

13. Cannon B and Nedergaard J: Brown fat tissue: Function and physiological significance. Physiol Rev 84: 277-359, 2004.

14. Lean ME: Brown adipose tissue in humans. Proc Nutr Soc 48: 243-256, 1989.

15. Huang YC, Chen TB, Hsu CC, Li SH, Wang PW, Lee BF, Kuo CY and Chiu NT: The relationship between brown adopse tissue activity and neoplastic status: An (18)F-FDG PET/CT study in the tropics. Lipids Health Dis 10: 238, 2011.

16. Ricquier D: Biology of brown adipose tissue: View from the chair. Int J Obes (Lond) 34 (Suppl 1): S3-S6, 2010.

17. Lecoultre V and Ravussin E: Brown adipose tissue and aging. Curr Opin Clin Nutr Metab Care 14: 1-6, 2011.

18. Shellock FG, Riedinger MS and Fishbein MC: Brown adopose tissue in cancer patients: Possible cause of cancer-induced cachexia. J Cancer Res Clin Oncol 111: 82-85, 1986.

19. Berstein LM: Cancer and heterogeneity of obesity: Apotential contribution of brown fat. Future Oncol 8: 1537-1548, 2012.

20. Sinha G: Homing in on the fat and caner connection. J Natl Cancer Inst 104: 966-967, 2012.

21. Cao Q, Hersl J, La H, Smith M, Jenkins J, Goloubeva O, Dilsizian V, Tkaczuk K, Chen W and Jones L: A pilot study of FDG PET/CT detects a link between brown adipose tissue and breast cancer. BMC Cancer 14: 126, 2014.

22. Allred DC, Harvey JM, Berardo M and Clark GM: Prognostic and predictive factors in breast cancer by immunohistochemical analysis. Mod Pathol 11: 155-168, 1998.

23. Shousha S: Oestrogen receptor status of breast carcinoma: Allred/H score conversion table. Histopathology 53: 346-347, 2008.

24. Truong MT, Erasmus JJ, Munden RF, Marom EM, Sabloff BS, Gladish GW, Podoloff DA and Macapinlac HA: Focal FDG uptake in mediastinal brown fat mimicking malignancy: A potential pitfall resolved on PET/CT. AJR 183: 1127-1132, 2004.

25. Hany TF, Gharehpapagh E, Kamel EM, Buck A, Himms-Hagen J and Von Schulthess GK: Brown adipose tissue: A factor to consider in symmetrical tracer uptake in the neck and upper chest region. Eur J Nucl Med Mol Imaging 29: 1393-1398, 2002.

26. Dobert N, Menzel C, Hamscho N, Wordehoff N, Kranert WT and Grünwald F: Atypical thoracic and supraclavicular FDG-uptake in paitents with Hodgkin's and non-Hodgkin's lymphoma. Q J Nucl Med Mol Imaging 48: 33-38, 2004.
27. Cinti S, Cancello R, Zingaretti MC, Ceresi E, De Matteis R, Giordano A, Himms-Hagen J and Ricquier D: CL316,243 and cold stress induce heterogeneous expression of UCP1 mRNA and protein in rodent brown adiposytes. J Histochem Cytochem 50: 21-31, 2001.

28. Lim S, Honek J, Xue Y, Seki T, Cao Z, Andersson P, Yang X, Hosaka K and Cao Y: Cold-induced activation of brown adipose tissue and adipose angiogenesis in mice. Nat Protoc 7: 606-615, 2012.

29. Kurozumi S, Matsumoto H, Hayashi Y, Tozuka K, Inoue K, Horiguchi J, Takeyoshi I, Oyama T and Kurosumi M: Power of PgR expression as a prognostic factor for ER-positive/HER2-negative breast cancer patients at intermediate risk classified by the Ki67 labeling index. BMC Cancer 17: 354, 2017.

30. Figueroa-Magalhães MC, Jelovac D, Connolly RM and Wolff AC: Treatment of HER2-positive breast cancer. Breast 23: 128-136, 2014.

31. Park J, Morley TS, Kim M, Clegg DJ and Scherer PE: Obesity and cancer-mechanisms underlying tumour progression and recurrence. Nat Rev Endocrinol 10: 455-465, 2014.

32. Howe LR, Subbaramaiah K, Hudis CA and Dannenberg AJ: Molecular pathways: Adipose inflammation as a mediator of obesity-associated cancer. Clin Cancer Res 19: 6074-6083, 2013.

33. Qiang L, Wang L, Kon N, Zhao W, Lee S, Zhang Y, Rosenbaum M, Zhao Y, Gu W, Farmer SR and Accili D: Brown remodeling of white adipose tissue by SirT1-dependent deacetylation of Ppar $\gamma$. Cell 150: 620-632, 2012.

34. Kourtidis A, Srinivasaiah R, Carkner RD, Brosnan MJ and Conklin DS: Peroxisome proliferator-activated receptor-gamma protects ERBB2-positive breast cancer cells from palmitate toxity. Breast Cancer Res 11: R16, 2009.

35. Tian L, Wang C, Hagen FK, Gormley M, Addya S, Soccio R, Casimiro MC,Zhou J,Powell MJ,Xu P, etal: Acetylation-defective mutants of Ppar $\gamma$ are associated with decreased lipid synthesis in breast cancer cells. Oncotarget 5: 7303-7315, 2014

36. Batista ML Jr, Peres SB, McDonald ME, Alcantara PS, Olivan M, Otoch JP, Farmer SR and Seelaender M: Adipose tissue inflammation and cancer cachexia: Possible role of nuclear transcription factors. Cytokine 57: 9-16, 2012

37. Rodriguez-Cuenca S, Monjo M, Roca P and Palou A: Opposite actions of testosterone and progesterone on UCP1 mRNA expression in cultured brown adipocytes. Cell Mol Life Sci 59: 1714-1723, 2002

38. Bartness TJ and Wade GN: Effects of interscapular brown adipose tissue denervation on body weight and energy metabolism in ovariectomized and estradiol-treated rats. Behav Neurosci 98: 674-685, 1984. 